\title{
Wound Healing and Skin Regeneration
}

\author{
Makoto Takeo $^{1,2}$, Wendy Lee ${ }^{1,2}$, and Mayumi Ito ${ }^{1,2}$ \\ ${ }^{1}$ The Ronald O. Perelman Department of Dermatology, New York University, School of Medicine, \\ New York, New York 10016 \\ ${ }^{2}$ Department of Cell Biology, New York University, School of Medicine, New York, New York 10016 \\ Correspondence: mayumi.ito@nyumc.org
}

The skin is a complex organ consisting of the epidermis, dermis, and skin appendages, including the hair follicle and sebaceous gland. Wound healing in adult mammals results in scar formation without any skin appendages. Studies have reported remarkable examples of scarless healing in fetal skin and appendage regeneration in adult skin following the infliction of large wounds. The models used in these studies have offered a new platform for investigations of the cellular and molecular mechanisms underlying wound healing and skin regeneration in mammals. In this article, we will focus on the contribution of skin appendages to wound healing and, conversely, skin appendage regeneration following injuries.

$T_{\mathrm{o}}^{\mathrm{h}}$ he skin is an intricate structure composed of the epidermis and dermis, including the subcutaneous fat, or dermal adipocyte layer. Environmental challenges to the barrier include penetration of harmful UV rays from the sun, invasion of harmful pathogens, and evaporation of water. Importantly, the skin also protects the underlying organs, a function necessary for the survival of the organism. As a protective shield for the body from the external environment, the skin is constantly exposed to potential injury, and thus wound healing is a vital process for the survival of all higher organisms. Epidermal appendages such as hair follicles, nails, and sweat glands help maintain and protect the skin and their important roles in wound healing continue to be elucidated. Better understanding of the cellular and molecular mechanisms un- derlying wound healing will ultimately allow us to influence and accelerate the wound repair/ regeneration process. This will benefit severe burn patients and amputees, especially in cases of extensive tissue loss and scarring.

Wound healing is a conserved evolutionary process among species and encompasses spatially and temporally overlapping processes including inflammation, blood clotting, and cellular proliferation and extracellular matrix (ECM) remodeling (Seifert et al. 2012b; Richardson et al. 2013). However, the outcome of wound healing in the skin differs between species. Some lower vertebrates including fish (zebrafish) and amphibians (axolotl and Xenopus) possess the ability to perfectly regenerate skin. It is known that after full-thickness excisional wounds in Xenopus froglets and axolotols, the

Editors: Anthony E. Oro and Fiona M. Watt

Additional Perspectives on The Skin and Its Diseases available at www.perspectivesinmedicine.org

Copyright (C) 2015 Cold Spring Harbor Laboratory Press; all rights reserved; doi: 10.1101/cshperspect.a023267

Cite this article as Cold Spring Harb Perspect Med 2015;5:a023267 
M. Takeo et al.

entire skin, including secretory appendages, regenerates (Yokoyama et al. 2011; Seifert et al. $2012 b)$. During this process, even the pigmentation pattern of the skin can be fully re-established (Seifert et al. 2012b). Zebrafish skin can also recover its striped pigmentation pattern following wounding, as well as regenerate subcutaneous adipocytes and scales during the healing process, making the regenerated skin almost indistinguishable from the original one (Richardson et al. 2013).

In contrast, it is challenging for adult mammals, including humans, to achieve such regeneration. Typically, wound healing in adult mammals results in scar tissue that lack skin appendages. Although scar formation can meet the requirements of the skin's fundamental function in preventing infection and dehydration, this process can also be unfavorable. Because of its obviously distinct appearance from the original intact skin, the scar formed as a result of injuries or burns can result in devastating cosmetic and psychological consequences, reducing the quality of life of the individual. Furthermore, skin appendages are an integral part of the skin's biological and physiological function. For example, skin epithelial appendages contribute epidermal cells for wound healing. Additionally, the hair follicle and sebaceous gland confer additional roles for the skin as sensory and thermoregulatory organs (Chen et al. 1997; Li et al. 2011). Consequently, scar formation prevents the complete recovery of skin function. Thus, the ability to restore the skin to its original state is highly valued. Interestingly, studies have reported remarkable examples of scarless healing in fetal skin and appendage regeneration in adult skin following the infliction of large wounds. The models used in these studies have offered a new platform for investigations of the cellular and molecular mechanisms underlying wound healing and skin regeneration in mammals. These may provide important insights into the regeneration of missing structures and redevelopment of fully functional skin. In this chapter, we will focus on the contribution of skin appendages to wound healing and, conversely, skin appendage regeneration following injuries.

\section{RE-EPITHELIALIZATION AND EPITHELIAL STEM CELLS}

The mammalian epidermis is a stratified squamous epithelium whose maintenance relies on proliferation and differentiation of the basal layer of the epidermis. As basal epidermal cells differentiate and move toward the surface, they give rise to suprabasal cells and the granular layer and eventually terminally differentiate into enucleated corneocytes composing the stratum corneum. As the outermost layer of the organism, the epidermis is constantly exposed to multiple forms of injury. Failure to re-epithelialize injured skin causes the loss of the barrier function of the organ, dehydration, infection or even death. Hence, rapid closure of the wound site by migration and proliferation of epithelial cells is critical to restore the barrier function that is vital for organism survival. A vast amount of evidence now shows that the presence and function of resident epithelial stem cells in adult skin fuel the re-epithelialization process.

Mascré et al. (2012) used two distinct promoters: Keratin14 that targets basal cells in the epidermis including a progenitor population that proliferates and differentiates, and Involculin that solely targets a committed progenitor cell population. Following wounding, both populations are recruited to the wound area but it is predominantly progeny of Keratin 14 expressing cells that survive long term, in contrast to progeny of Involculin expressing cells that were lost earlier. This study illustrates the importance of relatively undifferentiated cells in the basal layer of the skin epithelium, and their contribution to epidermal repair following injury.

Lineage tracing with mice that ubiquitously labels all keratinocytes of follicular origin (Shh-Cre;R26R-lacZ) showed that follicular cells can be converted to epidermal cells (Levy et al. 2007). Several distinct progenitor populations located in the hair follicle, including the bulge, upper bulge, hair follicle junctional zone next to the sebaceous gland and infundibulum have all been shown to contribute to the regenerating skin. Using label retaining techniques to trace the fate of slow cycling stem cells in the skin, 
Wound Healing and Skin Regeneration

hair follicle stem cells located in the bulge area of the hair follicle were shown to mobilize to the upper follicle (Taylor et al. 2000) and then to the skin epidermis after wounding (Tumbar et al. 2004). Consistent with these findings, transplantation studies have also shown that the bulge region from $\mathrm{R} 26-\mathrm{LaCZ}$ adult vibrissal follicles can contribute progenitors to reform the hair follicle, sebaceous gland, as well as the epidermis (Oshima et al. 2001). Subsequently, genetic tracing of Keratin 15 expressing epithelial stem cells located in the bulge/secondary hair germ region of the hair follicle showed that these cells migrate to the epidermis toward the center of the wound following full-thickness exisional wounds (Ito et al. 2005; Levy et al. 2005). Genetic ablation of $\mathrm{K}^{+} 5^{+}$cells does not result in defects in normal epidermal homeostasis suggesting that they only shift to epidermal cells in response to wounding. This illustrates that wounding perturbs epidermal homeostasis by cell depletion, resulting in recruitment of epithelial cells in the hair follicle that give rise to epidermal cells to promote re-epithelialization. Following migration to the epidermis, $\mathrm{K}^{+} 5^{+} \mathrm{ep}-$ ithelial stem cell progeny acquire an epidermal phenotype based on biochemical marker analysis. However, the observation that most of these cells disappear in the wound epidermis suggests that hair follicle bulge stem cells are primarily involved in the acute cell injury phase of wound repair (Fig. 1).

Lrig1 was the first marker identified for the junctional zone (isthmus) between the hair follicle bulge, sebaceous gland and infundibulum (Jensen et al. 2009). Lrig1 expressing cells can give rise to all adult epidermal lineages in skin reconstitution assays (Jensen et al. 2009). In contrast, more recent genetic labeling analysis revealed that Lrig $1^{+}$cells within the pilosebaceous unit contribute to neither the hair follicle nor the interfollicular epidermis, but solely the sebaceous gland or infundibulum during homeostasis (Page et al. 2013). After wounding, Lrig1 cells originating from the pilosebaceous compartment migrate to the wound area and persist up to 1 year in the regenerating IFE, and contribute permanently to the regenerating tissue following wounding (Page et al. 2013). Similarly, Lgr6-positive cells identified in the isthmus area are shown to contribute to reepithelialization (Snippert et al. 2010). Isolation of Lgr6-positive cells enabled them to reconstitute all of the epithelial lineages of the skin

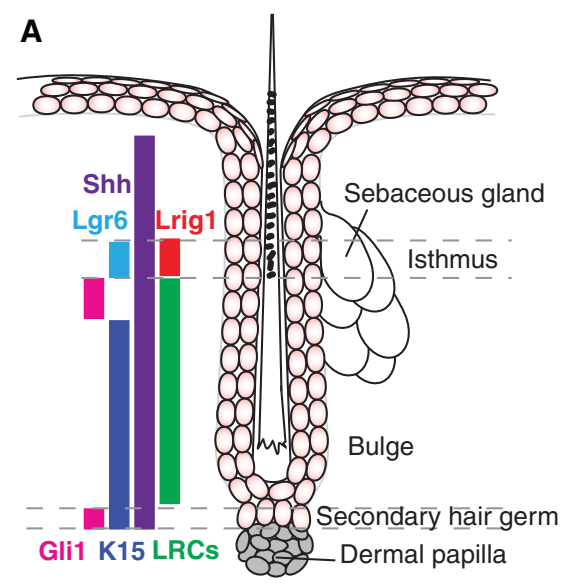

B

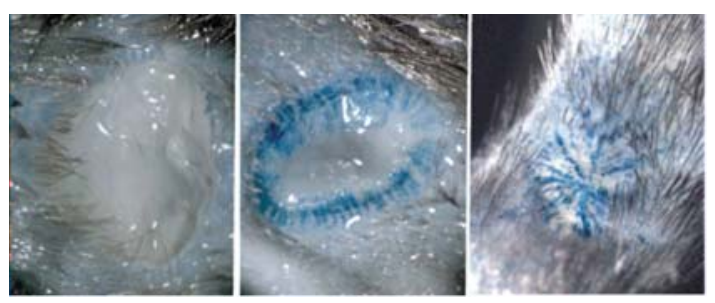

Figure 1. Contributions of hair follicle stem cells to re-epithelialization. (A) Schematic illustration of bulge stem cell markers that contribute to re-epithelialization. $(B)$ Lineage tracing of $\mathrm{K}_{1} 5^{+}$hair follicle stem cells after excisional wound using K15-LacZ mice. LacZ-positive cells were not found in IFE at $2 \mathrm{~d}$ after wounding (right panel). At $5 \mathrm{~d}, \mathrm{LacZ}^{+}$cells start to migrate from hair follicle to wound center (middle panel). At $8 \mathrm{~d}$ after wounding, re-epithelialization is completed and about $26 \%$ of re-epithelialized cells are lac $Z^{+}$(left panel). (From Ito et al. 2005; reprinted, with permission.) 
M. Takeo et al.

and form hair follicle bearing skin when combined with inductive dermal cells in transplantation assays. Genetic tracing showed that Lgr6 ${ }^{+}$ cells give rise to epidermis and participate in the hair follicle formation seen in the center of the wound. Lgr6 ${ }^{+}$cells persisted long term in the wound area suggesting that these cells have the ability to maintain or reacquire the self-renewing capacity following exit from their original niche within the isthmus. These studies establish that progenitors residing in compartments other than the bulge including the isthmus and junctional zone convert into self-sustaining epidermal stem cells in response to trauma. Furthermore, the study by Brownell et al. (2011) showed that $\mathrm{Glil}^{+}$upper bulge cells contribute to the epidermal healing to establish long-term progenitors following wounding. Interestingly, following skin denervation, $\mathrm{Gli}^{+}$cells can still contribute to the initial re-epithelialization but they are not maintained in the regenerated epidermis. These results suggest that $\mathrm{Gli1}^{+} \mathrm{K} 15^{-}$ upper bulge cells depend on a perineural stem cell niche for their ability to adopt epidermal stem cell fate after wounding. These studies suggest that extrinsic niche derived signals may determine the ability of follicular keratinocytes to become long-term epidermal progenitors after migrating from the hair follicle. Currently, it is not known how much the re-epithelialized area is occupied by epithelial stem cells nor how they are patterned in the re-epithelialized layer. Understanding these may provide a platform to investigate mechanisms of how cells from distinct lineages show different persistence in the new epidermis. Moreover, understanding the extent of how the adult skin epidermis is capable of regenerating stem cells will define whether reepithelialization in wounded mammals represents the true regeneration process.

Although these studies established that hair follicle epithelial stem cells contribute to reepithelialization, a recurring question has been whether the hair follicle is necessary for wound healing of hair bearing skin. A study that experimentally addressed this entailed wounding Edaradd ${ }^{\text {cr } / \text { cr }}$ mice that lack hair follicles in the tail skin (Langton et al. 2008). They showed that the tail skin of these mutant mice do not close their wounds as efficiently as control tail skin containing hair follicles. However, after an initial lag period, the wound re-epithelializes at the same rate. From these results, it was concluded that hair follicle cells accelerate the onset of wound healing but are not necessary for wound healing. The relevance of hair follicles to wound healing was also shown by a study that investigated the influence of hair cycle stages on the rate of wound healing. Ansell et al. (2011) showed that wound-healing rates were different depending on the hair cycle stage, in which wound healing was faster, during the anagen phase of hair follicle cycling in vivo. This was likely caused by the extensive blood vessel network, relative immunosuppression, reduction in cell adhesion genes, and increase in developmental pathway genes by follicular epithelial cells during the anagen stage compared with the telogen stage. This is consistent with another study that showed that anagen hair follicles produce angiogenic factors (Mecklenburg et al. 2000). More recently, it was shown that mice deficient for the proapoptotic Sept4/ARTS gene have elevated numbers of hair follicle stem cells as a result of reduced apoptosis (Fuchs et al. 2013), which normally occurs in the catagen phase (Ito et al. 2004). Sept4/ARTS - / - mice display significant improvement in wound healing. Furthermore, this phenotype depended on K15-expressing hair follicle stem cells as indicated by lineage tracing.

Taken together, these studies illustrate the vital function of hair follicles as a cellular reservoir for healing skin and as a signaling center that affects the behavior of nonhair follicle cells.

\section{SCARLESS WOUND-HEALING PROCESS IN MAMMALS}

In association with re-epithelialization, the restoration of dermis takes place by migration and proliferation of fibroblasts. The response of fibroblasts during wound healing determines the outcome of tissue repair. In response to wounding, macrophages and fibroblasts release growth factors that lead to further fibroblast migration and proliferation. They also release inflammatory cytokines to induce the immune response 
to protect against external pathogens in the wound. These fibroblasts also produce collagens and other extracellular matrix proteins to aid in wound repair. While collagen and ECM deposition are required to close the wound efficiently, they are also deleteriously responsible for fibrosis and scarring of the skin. Recent studies have shown an essential role for intradermal adipocytes in fibroblast activation and migration (Schmidt and Horsley 2013), suggesting that multiple cell types in the dermis function to activate the fibroblast proliferation and migration necessary to repair the dermis. Recently, transplantation and lineage tracing have identified two distinct dermal lineages that give rise to the upper and lower dermis, respectively. Following wounding, both cell types are recruited to the wounded area. One population forms the lower dermis in the initial injury response. In contrast, the progeny that gives rise to the upper dermis is recruited during re-epithelialization and provides an environment for new hair follicle formation in the wounded area. This study illustrates the importance of the dermal contribution to not only wound healing, but also to appendage regeneration (Driskell et al. 2013).

In 1970, a seminal article reported that rabbit fetuses can heal wounds without the appearance of scar formation (Somasundaram and Prathap 1970). Since then, similar observations have been reported in other mammals, including sheep, mice, rats, and humans (Somasundaram and Prathap 1970; Burrington 1971; Sopher 1972; Rowlatt 1979; Hallock 1985). In Somasundaram and Prathap's study (Hallock 1985), a $0.5-\mathrm{cm}$ disc of skin of either newborn or fetal (14-25 d after gestation) rabbits was excised. In newborn rabbits, wound contraction and scab formation are observed by 6 days after wounding, resulting in granulation tissue development and scar formation. In contrast, a compact layer of spindle-shaped cells, two to three cells thick, initially covered the fetal wound surface. There was no apparent sign of wound contraction, subsequent granulation tissue development, or scar formation. These pioneering studies did not describe or discuss whether and how skin appendage regeneration accompanied the scarless wound healing, presumably because of limitations in distinguishing between embryonic development processes of skin appendages and wound-induced de novo regeneration of skin appendages at that time.

Since then, scar-free wound healing of human fetuses was reported in 1979 (Rowlatt 1979), and subsequent efforts have been directed at investigating the mechanisms underlying scar-free wound healing by comparing the wound-healing processes between scarless and scarring wounds in multiple animal models. A key difference identified in fetal wound healing is a low inflammatory reaction owing to the absence of a fully developed immune system. In scarless wounds, neutrophils, macrophages, and mast cells have differences in size and maturity when compared with scarring wounds (Satish and Kathju 2010; Wulff et al. 2012). Intriguingly, Urodeles such as newts, capable of perfectly regenerating multiple organs including the skin and limb, are immunodeficient compared with other amphibians such as Xenopus that show a more limited ability to regenerate (reviewed by Cohen 1971). The correlation between the immune system and competence for regeneration led to a traditional hypothesis in wound-healing studies: inflammation may restrict regeneration by promoting fibrosis and scar formation.

The inflammatory response marks one of the earliest responses in the adult skin to wounding stimuli, and a number of studies have shown that immune cell infiltration and signaling play a key role in scar formation and fibrosis. Immune cells and macrophages accumulate at a wound site not only to fight against invading microorganisms but also to produce various growth factors such as FGFs and TGF- $\beta$ to guide re-epithelialization, fibroblast repopulation and ECM remodeling. Therefore it is plausible that scarring or fibrosis during skin repair is promoted by cytokines or growth factors produced by the inflammatory response. However, a similar cocktail of growth factors sufficient to promote scar formation may also be provided by skin resident cells even in the absence of a well-developed immune system. In contrast to the seminal work by Barbul et al., which showed that immunodeficient Foxn1 null adult mice that 
M. Takeo et al.

lack T lymphocytes close wounds without scar formation (Barbul et al. 1989), subsequent studies in other immunodeficient mouse models, such as Rag1-deficient mice and SCID (severe combined immune deficiency) mice, which lack both $\mathrm{B}$ and $\mathrm{T}$ lymphocytes, showed that wounds on these mice heal with scar formation (Gawronska-Kozak et al. 2006). Similarly, it is reported that thymus-depleted mice and mice treated with immunosuppressants also heal with scar formation (Gawronska-Kozak et al. 2006). These results suggest that scar formation is not merely dependent on the degree of the lymphocyte-mediated inflammatory response to wounding.

In efforts to identify the distinct molecular characteristics in scarless wound healing, Dang et al. (2003) analyzed the expression of matrix metalloproteinases (MMP) and their inhibitor TIMPs in scarless wounds and scarring wounds in rats. They made full-thickness excisional wounds on the dorsum of E16 and E19 fetal rats in which wounds heal without or with scars, respectively. They compared gene expression of MMPs and TIMPs at 24, 48, and $72 \mathrm{~h}$ after wounding and found that scarless wounds have greater MMP expression relative to TIMPs in wounds that heal with a scar. More recently, Colwell et al. made excisional wounds on the dorsal back skin of E17 fetal mice and 3-wkold postnatal mice, respectively. They compared gene expression profiles between scarless fetal wounds and scarring postnatal wounds by microarray at 1,12 , and $24 \mathrm{~h}$, and found that upregulation of genes involved in DNA transcription and repair, cell-cycle regulation, protein homeostasis, and intracellular signaling is detected more rapidly in response to wounding in fetal wounds (Colwell et al. 2008). Additionally, comparisons of scarless fetal wounds and adult wounds report that one of the most prominent and consistent differences between the two is the high expression of TGF- $\beta 3$ in mouse and rat fetus (Cowin et al. 2001; Soo et al. 2003). Shah et al. showed that administration of exogenous TGF- $\beta 3$ into the dermis at the margins of a full-thickness wound for 3 days reduced monocyte and macrophage accumulation in the wound area, resulting in reduced scar for- mation with decreased fibronectin and collagen I and III deposition in the early stages of wound healing (Shah et al. 1995). These studies suggest that production of TGF- $\beta 3$ may be a key factor to achieving scar-free wound healing. These basic animal studies pave the way to clinical trials to control scar formation in humans, which show that injection of recombinant human TGF- $\beta 3$ after injury or surgical removal of scar tissue significantly reduces scar formation (Occleston et al. 2008; So et al. 2011). Despite these advancements, there is still no treatment that completely prevents scar formation and induces regeneration of skin appendages including hair follicles.

\section{REGENERATION OF SKIN APPENDAGES- HAIR FOLLICLE NEOGENESIS}

Several of the molecular and cellular events that orchestrate mammalian wound healing have been elucidated over the past several years. However, there is still a lack of therapeutic interventions that lead to perfect scarless skin regeneration that includes appendages in the adult skin. Research aimed to influence the wound repair process to promote regenerative healing requires experimental models in which molecular and cellular interactions can be efficiently dissected. Previous studies established that de novo hair follicles form in the wound area by recapitulating embryonic hair follicle development, illustrating the remarkable regenerative capacity of the adult skin (Ito et al. 2007). Moreover, this phenomenon occurred in normal wild-type mice and therefore serves as a powerful model to study how growth and patterning mechanisms can be properly activated and used to regenerate missing appendages.

Hair follicle formation typically only occurs during embryonic development under homeostatic conditions. During embryonic development, cellular interactions between epithelial and mesenchymal cells lead to the formation of a hair placode and dermal papilla, and their reciprocal interactions lead to morphogenesis and growth of the hair follicle. Coordinated activation of several key signaling pathways including Wnt $/ \beta$-catenin and BMP regulators is 


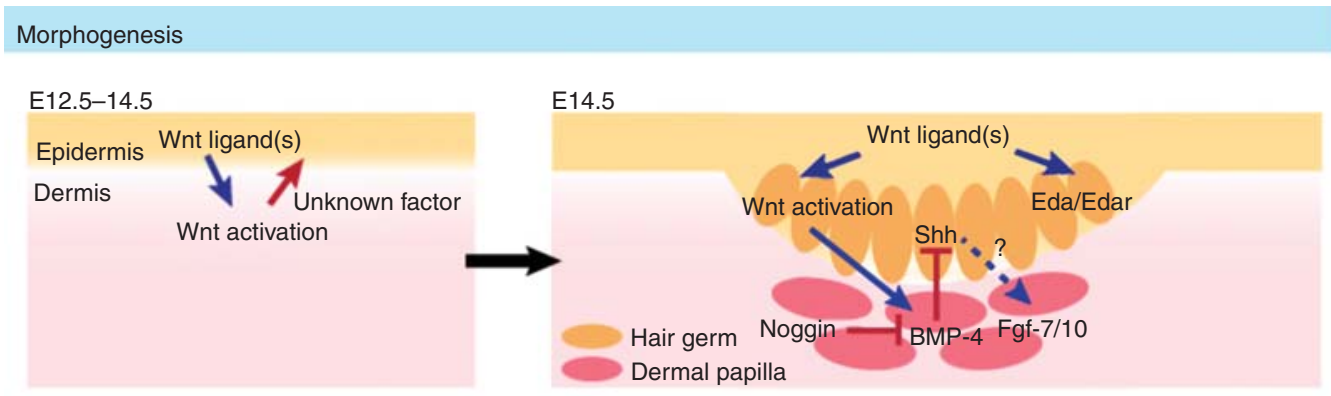

Hair follicle neogenesis

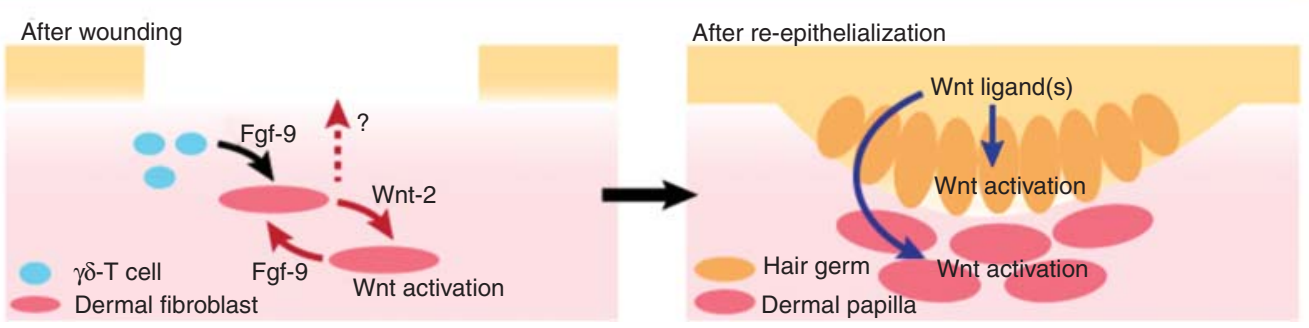

Figure 2. Schematic illustration of molecular mechanisms during hair follicle morphogenesis (top) and neogenesis (bottom).

essential for this process (Fig. 2) (Millar 2002; Schmidt-Ullrich and Paus 2005; Myung and Ito 2012; Sennett and Rendl 2012). Once the hair follicle forms, hair is produced cyclically by interactions between epithelial stem cells in the hair follicle bulge/secondary hair germ area and dermal papilla cells throughout life (Cotsarelis et al. 1990; Kishimoto et al. 2000; Botchkarev et al. 2001; Ito et al. 2002, 2004; Rendl et al. 2008; Greco et al. 2009; Zhang et al. 2009; Enshell-Seijffers et al. 2010; Garza et al. 2011; Clavel et al. 2012; Oshimori and Fuchs 2012; Myung et al. 2013).

Mouse models with small punch wounds $(\sim 6 \mathrm{~mm}$ ) heal largely by contraction (note that contraction accounts for $\sim 90 \%$ of repair of mouse skin), while hair follicles fail to regenerate in the wound site (Ito et al. 2007). In contrast, after a large wound is made on the back skin $\left(1 \mathrm{~cm}^{2}\right.$ in $3 \mathrm{wk}$ old, or $2.25 \mathrm{~cm}^{2}$ in $<7 \mathrm{wk}$ old), contraction stops before wound closure leaving a measurable wound scar area. Re-epithelialization of large wounds occurs approximately 2 wk after wounding in adult mice. Remarkably, although there is a lack of hair follicles in the wound area at the completion of re-epithelialization, de novo hair follicles are found in this area $2-3 \mathrm{~d}$ after re-epithelialization. It is unknown how hair follicle neogenesis occurs only in the center of the wounds and is absent in the peripheral area of wounds. During hair follicle neogenesis, expression of several genes that are required for embryonic hair follicle development, such as Wnt10b, Lef1, and Shh, were observed. Moreover, in transgenic mice in which epithelial cells secrete the Wnt inhibitor, Dkk1, wound closure occurs normally, while hair neogenesis is inhibited. Similar defects were also observed in another transgenic mouse that lacks epithelial Wntless (Wls) expression, which is required for Wnt ligand secretion (Myung et al. 2013). In contrast, the number of regenerated hair germs significantly increased in K14Wnt7a transgenic mice that overexpress Wnt7a in the epidermis. These results suggest that the secretion of Wnt ligands from the epidermis promotes hair follicle neogenesis in adult mice (Fig. 2).

A more recent study from Gay et al. (2013) showed the role of FGF9 secretion from $\gamma \delta$-T 
M. Takeo et al.

cells and activation of Wnt signaling in dermal fibroblasts on hair follicle neogenesis (Fig. 2). They found that following large full-thickness excisional wounds, $\gamma \delta$-T immune cells accumulate within the wound site and secrete FGF9 before re-epithelialization. Subsequently, FGF-9 induces Wnt2 expression in dermal fibroblasts, which in turn leads to activation of Wnt signaling in dermal fibroblast in an autocrine manner and induces FGF-9 expression in Wnt-activated dermal fibroblasts. In mice that lack $\gamma \delta$-T cells ( $\mathrm{Tcrb}^{-/-}$mice ) or FGF-9 expression specifically in T cells (Lck-Cre:Fgff ${ }^{f l / f l}$ mice), wound closure occurs normally, while Wnt signaling is abrogated in dermal fibroblasts, resulting in decreased hair neogenesis. These findings showed that the interplay between immune cells and skin fibroblasts recruited to the wound site is essential to the activation of molecular pathways that promote hair follicle regeneration. On the other hand, another study showed that application of prostaglandin $\left(\mathrm{PGD}_{2}\right)$, an inflammatory mediator, inhibits hair neogenesis while new hair formation is enhanced in mice that lack a receptor for $\mathrm{PGD}_{2}$, GPR44 (Nelson et al. 2013). These results suggest that inflammatory mediators involved in wound healing have the ability to modulate hair follicle regeneration in the wound area.

Although de novo hair follicles formed in the wound were repopulated with functional epithelial stem cells that possess the ability to drive cyclical growth of the new hair follicles, most of the neogenic hairs were unpigmented (Ito et al. 2007; Chou et al. 2013). It is believed that formation of unpigmented hair follicle was caused by the lack of melanocytes in the wound area. In the normal skin, melanocyte stem cells (McSCs), responsible for hair pigmentation, are located in the hair follicle bulge and secondary hair germ niche together with epithelial stem cells (Nishimura et al. 2002). The coordinated activation of these two stem cell populations through co-activation of Wnt signaling results in pigmented hair production (Rabbani et al. 2011). McSCs in the hair follicles that surround the wound migrate upward and populate the wound epidermis (Fig. 3) (Chou et al. 2013). It was also found that the distribution of epi- dermal melanocytes is restricted to the wound periphery, and epidermal melanocytes are occasionally found in the wound center where hair neogenesis occurs. In instances where melanocytes are able to migrate to the center, epidermal melanocytes participate in hair neogenesis. As de novo hair follicles develop, McSCs are re-established in the newly formed bulge, and these hair follicles produce pigmented hair. These results indicate that when melanocytes are present in the region of the skin where de novo hair follicle formation occurs, these cells can properly interact with epidermal and dermal cells to participate in hair follicle neogenesis after wounding.

Recently, similar hair follicle neogenesis was reported in another mouse strain, the African spiny mouse (Acomys) (Seifert et al. 2012a). Sixty percent of the total dorsal surface area of these mice are torn up as a result of autotomy and regenerate pigmented hair follicles within $30 \mathrm{~d}$ after autotomy. Acomys can also regenerate hair follicles after $4 \mathrm{~mm}^{2}$ small wounds unlike other mice. Moreover, the wound-healing process of Acomys shared similar characteristics observed in mammalian fetuses, such as slow deposition of the extracellular matrix and high-level expression of collagen III. These observations suggest that hair follicle neogenesis may occur more effectively when the woundhealing process is similar to what occurs in fetuses.

\section{CONCLUDING REMARKS AND REMAINING QUESTIONS}

Previous studies have established the contribution of skin appendages to wound healing. How wounding stimuli recruit stem cells for re-epithelialization is not fully understood. Further understanding of the molecular mechanisms underlying the plasticity of epithelial stem cells in the hair follicle will be necessary to design strategies to efficiently exploit stem cells to enhance tissue regeneration during wound healing. At the same time, it is now clear that wound stimuli can trigger the skin to engage embryonic programs to regenerate hair follicles in adult mice. The relationship between scarless heal- 

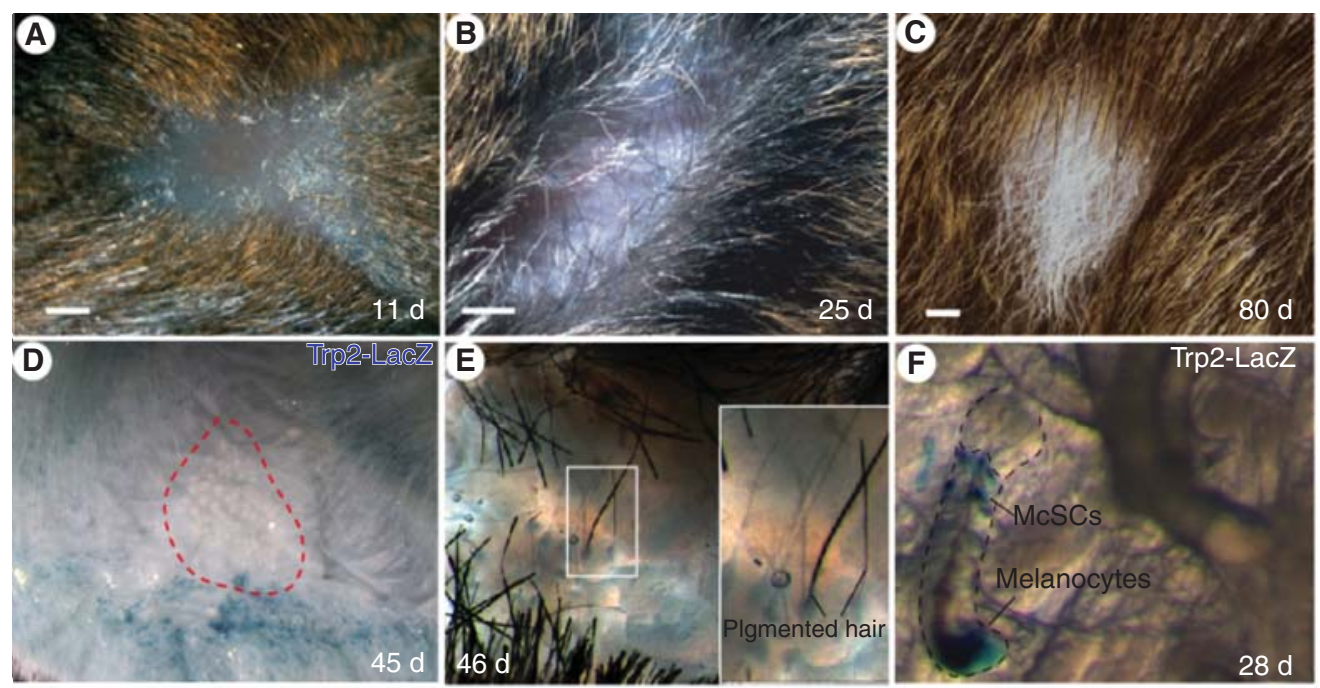

G Wound periphery

Wounded site

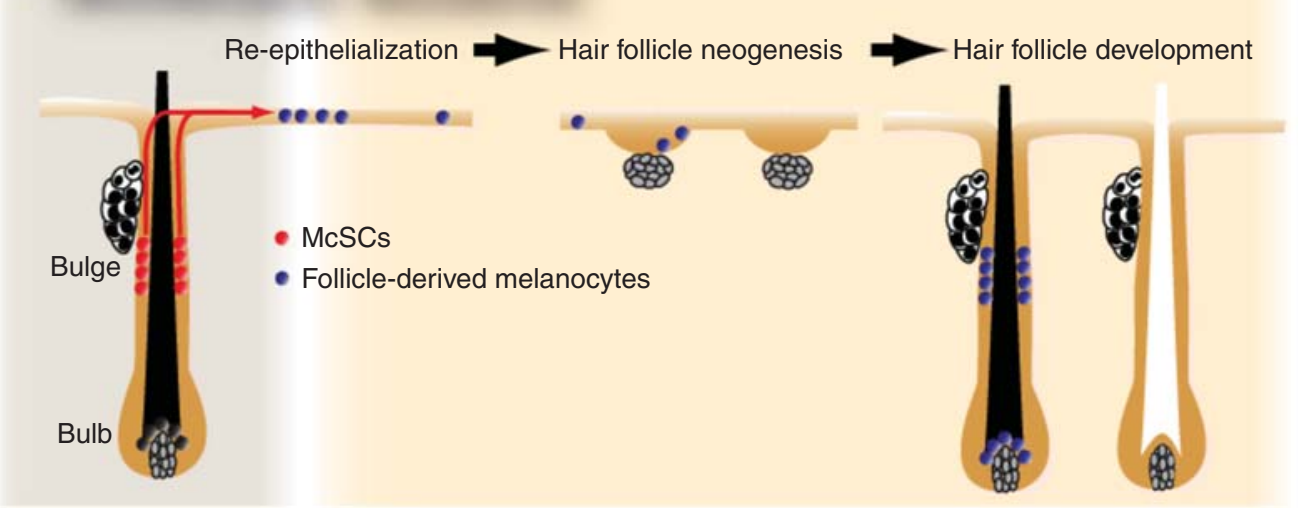

Figure 3. Contribution of hair follicle-derived melanocytes to hair follicle neogenesis. $(A-C)$ Hair regeneration from newly formed hair follicle in wound area. Note that almost all hair lack pigment. $(D)$ Distribution of hair follicle derived melanocytes in the wound in Dct-LacZ reporter mice in which melanocyte including McSCs are labeled with LacZ. Most of the epidermal melanocytes are restricted to the wound periphery, and only a few melanocytes are found in the center of wound where hair follicle neogenesis occurs (red dashed line). $(E)$ Occasional pigmented hair regeneration. $(F)$ Whole mount view of newly formed hair follicle that produce pigmented hair. Note the presence of McSCs in the bulge. $(G)$ Schematic illustration of the contribution of hair follicle-derived melanocytes to hair follicle neogenesis. Scale bars, $1 \mathrm{~mm}$. ( $A-C$ from Ito et al. 2007; reproduced, with permission; $D-G$ from Chou et al. 2013; reproduced, with permission.)

ing and the ability to regenerate epidermal appendages in the wound site is currently unclear. Wound-induced scar formation and epidermal appendage regeneration likely share some common signals or mechanisms. However, it is unknown how the mechanisms that lead to these two distinct consequences of wound healing are synchronized. For example, it is still unclear whether precluding scar formation is a prerequisite to permit the skin to regenerate its appendage or whether the addition of morphogenetic signals in the presence of scar formation can promote epidermal appendage formation. To integrate our understanding of wound healing and appendage regeneration, it will be important to dissect how molecular signals that 
M. Takeo et al.
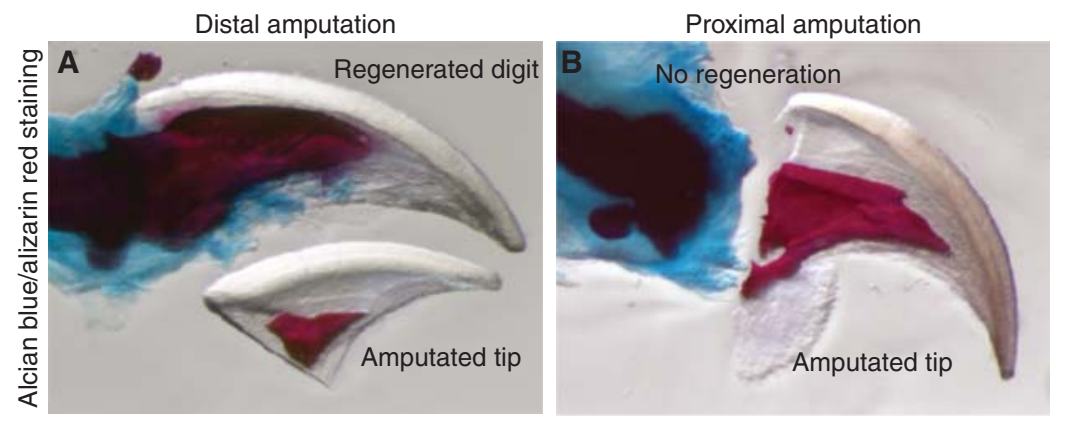

Figure 4. Relationship between nail regeneration and digit regeneration. Alcian blue/alizarin red staining of mouse digit at 5 weeks after distal $(A)$, or proximal $(B)$ amputation. When a digit is amputated at the distal level, both nail and underlying mesenchymal digit bone regenerates $A$, while neither nail nor digit bone regenerates from proximal amputation $B$. (Panel $B$ is from Takeo et al. 2013; reproduced, with permission.)

are activated at each stage of the wound-healing process influence the behavior of each skin cell type and how these signals ultimately promote scarring and/or appendage regeneration.

Interestingly, epidermal appendage regeneration also impacts surrounding cells. This concept is illustrated by a study that showed that epithelial stabilization of $\beta$-catenin in adult skin, known to trigger de novo hair follicle formation, confers embryonic characteristics to surrounding dermal cells (Collins et al. 2011). These observations suggest that epidermal appendage formation is not merely a result of regenerative healing, but may be allowing regenerative healing of surrounding dermal cells. Importantly, on amputation of the mouse digit tip, regeneration of underlying mesenchymal bone occurs only in association with nail regeneration (Fig. 4) (Borgens 1982; Zhao and Neufeld 1995; Mohammad et al. 1999; Takeo et al. 2013). Wound healing following digit amputations proximal to the visible nail plate lacks nail regeneration and ends with scarring. These studies highlight the possibility that epidermal appendage formation emits multiple morphogens that signal to other cell types in a paracrine manner. Little is known about the interplay between heterotypic cells that coordinately orchestrate wound healing and regeneration. Dissecting the molecular crosstalk between epidermal appendage regeneration and dermal repair may provide important clues to instruct dermal cells to engage embryonic/regenerative programs and reduce scarring. These future studies hold the promise to develop novel and innovative approaches to exploit our cumulative understanding of signals that govern epidermal appendage regeneration and wound healing.

\section{ACKNOWLEDGMENTS}

W.L. is supported by the NYU Kimmel Stem Cell Center and NYSTEM training Grant C026880. M.I. is supported by the U.S. National Institutes of Health, National Institute of Arthritis and Musculoskeletal and Skin Diseases Grant 1R01AR59768-01A1, and the Ellison Medical Foundation.

\section{REFERENCES}

Ansel DM, Kloepper JE, Thomason HA, Paus R, Hardman MJ. 2011. Exploring the "hair growth-wound healing connection": Anagen phase promotes wound re-epithelialization. J Invest Dermatol 131: 518-528.

Barbul A, Shawe T, Rotter SM, Efron JE, Wasserkrug HL, Badawy SB. 1989. Wound healing in nude mice: A study on the regulatory role of lymphocytes in fibroplasia. Surgery 105: 764-769.

Borgens RB. 1982. Mice regrow the tips of their foretoes. Science 217: 747-750.

Botchkarev VA, Botchkareva NV, Nakamura M, Huber O, Funa K, Lauster R, Paus R, Gilchrest BA. 2001. Noggin is required for induction of the hair follicle growth phase in postnatal skin. FASEB J 15: 2205-2214.

Brownell I, Guevara E, Bai CB, Loomis CA, Joyner AL. 2011. Nerve-derived sonic hedgehog defines a niche for hair follicle stem cells capable of becoming epidermal stem cells. Cell Stem Cell 8: 552-565. 
Wound Healing and Skin Regeneration

Burrington JD. 1971. Wound healing in the fetal lamb. $J$ Pediatr Surg 6: 523-528.

Chen W, Kelly MA, Opitz-Araya X, Thomas RE, Low MJ, Cone RD. 1997. Exocrine gland dysfunction in MC5-Rdeficient mice: Evidence for coordinated regulation of exocrine gland function by melanocortin peptides. Cell 91: 789-798.

Chou WC, Takeo M, Rabbani P, Hu H, Lee W, Chung YR, Carucci J, Overbeek P, Ito M. 2013. Direct migration of follicular melanocyte stem cells to the epidermis after wounding or UVB irradiation is dependent on Mclr signaling. Nat Med 19: 924-929.

Clavel C, Grisanti L, Zemla R, Rezza A, Barros R, Sennett R, Mazloom AR, Chung CY, Cai X, Cai CL, et al. 2012. Sox2 in the dermal papilla niche controls hair growth by finetuning BMP signaling in differentiating hair shaft progenitors. Dev Cell 23: 981-994.

Cohen N. 1971. Amphibian transplantation reactionsReview. Am Zool 11: 193.

Collins CA, Kretzschmar K, Watt FM. 2011. Reprogramming adult dermis to a neonatal state through epidermal activation of $\beta$-catenin. Development 138: 5189 5199.

Colwell AS, Longaker MT, Peter Lorenz H. 2008. Identification of differentially regulated genes in fetal wounds during regenerative repair. Wound Repair Regen 16: 450-459.

Cotsarelis G, Sun TT, Lavker RM. 1990. Label-retaining cells reside in the bulge area of pilosebaceous unit: Implications for follicular stem cells, hair cycle, and skin carcinogenesis. Cell 61: 1329-1337.

Cowin AJ, Holmes TM, Brosnan P, Ferguson MW. 2001 Expression of TGF- $\beta$ and its receptors in murine fetal and adult dermal wounds. Eur J Dermatol 11: 424-431.

Dang CM, Beanes SR, Lee H, Zhang X, Soo C, Ting K. 2003. Scarless fetal wounds are associated with an increased matrix metalloproteinase-to-tissue-derived inhibitor of metalloproteinase ratio. Plast Reconstr Surg 111: 22732285.

Driskell RR, Lichtenberger BM, Hoste E, Kretzschmar K, Simons BD, Charalambous M, Ferron SR, Herault Y, Pavlovic G, Ferguson-Smith AC, et al. 2013. Distinct fibroblast lineages determine dermal architecture in skin development and repair. Nature 504: 277-281.

Enshell-Seijffers D, Lindon C, Kashiwagi M, Morgan BA. 2010. $\beta$-Catenin activity in the dermal papilla regulates morphogenesis and regeneration of hair. Dev Cell 18: 633-642.

Fuchs Y, Brown S, Gorenc T, Rodriguez J, Fuchs E, Steller H. 2013. Sept4/ARTS regulates stem cell apoptosis and skin regeneration. Science 341: 286-289.

Garza LA, Yang CC, Zhao T, Blatt HB, Lee M, He H, Stanton DC, Carrasco L, Spiegel JH, Tobias JW, et al. 2011. Bald scalp in men with androgenetic alopecia retains hair follicle stem cells but lacks CD200-rich and CD34-positive hair follicle progenitor cells. J Clin Invest 121: 613-622.

Gawronska-Kozak B, Bogacki M, Rim JS, Monroe WT, Manuel JA. 2006. Scarless skin repair in immunodeficient mice. Wound Repair Regen 14: 265-276.

Gay D, Kwon O, Zhang Z, Spata M, Plikus MV, Holler PD, Ito M, Yang Z, Treffeisen E, Kim CD, et al. 2013. Fgf9 from dermal $\gamma \delta$-T cells induces hair follicle neogenesis after wounding. Nat Med 19: 916-923.

Greco V, Chen T, Rendl M, Schober M, Pasolli HA, Stokes N, Dela Cruz-Racelis J, Fuchs E. 2009. A two-step mechanism for stem cell activation during hair regeneration. Cell Stem Cell 4: 155-169.

Hallock GG. 1985. In utero cleft lip repair in A/J mice. Plast Reconstr Surg 75: 785-790.

Ito M, Kizawa K, Toyoda M, Morohashi M. 2002. Labelretaining cells in the bulge region are directed to cell death after plucking, followed by healing from the surviving hair germ. J Invest Dermatol 119: 1310-1316.

Ito M, Kizawa K, Hamada K, Cotsarelis G. 2004. Hair follicle stem cells in the lower bulge form the secondary germ, a biochemically distinct but functionally equivalent progenitor cell population, at the termination of catagen. Differentiation 72: 548-557.

Ito M, Liu Y, Yang Z, Nguyen J, Liang F, Morris RJ, Cotsarelis G. 2005. Stem cells in the hair follicle bulge contribute to wound repair but not to homeostasis of the epidermis. Nat Med 11: 1351-1354.

Ito M, Yang Z, Andl T, Cui C, Kim N, Millar SE, Cotsarelis G. 2007. Wnt-dependent de novo hair follicle regeneration in adult mouse skin after wounding. Nature 447: 316320.

Jensen KB, Collins CA, Nascimento E, Tan DW, Frye M, Itami S, Watt FM. 2009. Lrig1 expression defines a distinct multipotent stem cell population in mammalian epidermis. Cell Stem Cell 4: 427-439.

Kishimoto J, Burgeson RE, Morgan BA. 2000. Wnt signaling maintains the hair-inducing activity of the dermal papilla. Genes Dev 14: 1181-1185.

Langton AK, Herrick SE, Headon DJ. 2008. An extended epidermal response heals cutaneous wounds in the absence of a hair follicle stem cell contribution. J Invest Dermatol 128: 1311-1318.

Levy V, Lindon C, Harfe BD, Morgan BA. 2005. Distinct stem cell populations regenerate the follicle and interfollicular epidermis. Dev Cell 9: 855-861.

Levy V, Lindon C, Zheng Y, Harfe BD, Morgan BA. 2007. Epidermal stem cells arise from the hair follicle after wounding. FASEB J 21: 1358-1366.

Li L, Rutlin M, Abraira VE, Cassidy C, Kus L, Gong S, Jankowski MP, Luo W, Heintz N, Koerber HR, et al. 2011. The functional organization of cutaneous lowthreshold mechanosensory neurons. Cell 147: 16151627.

Mascré G, Dekoninck S, Drogat B, Youssef KK, Brohee S, Sotiropoulou PA, Simons BD, Blanpain C. 2012. Distinct contribution of stem and progenitor cells to epidermal maintenance. Nature 489: 257-262.

Mecklenburg L, Tobin DJ, Muller-Rover S, Handjiski B, Wendt G, Peters EM, Pohl S, Moll I, Paus R. 2000. Active hair growth (anagen) is associated with angiogenesis. J Invest Dermatol 114: 909-916.

Millar SE. 2002. Molecular mechanisms regulating hair follicle development. J Invest Dermatol 118: 216-225.

Mohammad KS, Day FA, Neufeld DA. 1999. Bone growth is induced by nail transplantation in amputated proximal phalanges. Calcif Tissue Int 65: 408-410. 
M. Takeo et al.

Myung P, Ito M. 2012. Dissecting the bulge in hair regeneration. J Clin Invest 122: 448-454.

Myung PS, Takeo M, Ito M, Atit RP. 2013. Epithelial Wnt ligand secretion is required for adult hair follicle growth and regeneration. J Invest Dermatol 133: 31-41.

Nelson AM, Loy DE, Lawson JA, Katseff AS, Fitzgerald GA, Garza LA. 2013. Prostaglandin $\mathrm{D}_{2}$ inhibits wound-induced hair follicle neogenesis through the receptor, Gpr44. J Invest Dermatol 133: 881-889.

Nishimura EK, Jordan SA, Oshima H, Yoshida H, Osawa M, Moriyama M, Jackson IJ, Barrandon Y, Miyachi Y, Nishikawa S. 2002. Dominant role of the niche in melanocyte stem-cell fate determination. Nature 416: 854-860.

Occleston NL, Laverty HG, O'Kane S, Ferguson MW. 2008. Prevention and reduction of scarring in the skin by transforming growth factor $\beta 3$ (TGF- $\beta 3$ ): From laboratory discovery to clinical pharmaceutical. J Biomater Sci Polym Ed 19: 1047-1063.

Oshima H, Rochat A, Kedzia C, Kobayashi K, Barrandon Y. 2001. Morphogenesis and renewal of hair follicles from adult multipotent stem cells. Cell 104: 233-245.

Oshimori N, Fuchs E. 2012. Paracrine TGF- $\beta$ signaling counterbalances BMP-mediated repression in hair follicle stem cell activation. Cell Stem Cell 10: 63-75.

Page ME, Lombard P, Ng F, Gottgens B, Jensen KB. 2013. The epidermis comprises autonomous compartments maintained by distinct stem cell populations. Cell Stem Cell 13: 471-482.

Rabbani P, Takeo M, Chou W, Myung P, Bosenberg M, Chin L, Taketo MM, Ito M. 2011. Coordinated activation of Wnt in epithelial and melanocyte stem cells initiates pigmented hair regeneration. Cell 145: 941-955.

Rendl M, Polak L, Fuchs E. 2008. BMP signaling in dermal papilla cells is required for their hair follicle-inductive properties. Genes Dev 22: 543-557.

Richardson R, Slanchev K, Kraus C, Knyphausen P, Eming S, Hammerschmidt M. 2013. Adult zebrafish as a model system for cutaneous wound-healing research. J Invest Dermatol 133: 1655-1665.

Rowlatt U. 1979. Intrauterine wound healing in a 20 week human fetus. Virchows Arch A Pathol Anat Histol 381: 353-361.

Satish L, Kathju S. 2010. Cellular and molecular characteristics of scarless versus fibrotic wound healing. Dermatol Res Pract 2010: 790234.

Schmidt BA, Horsley V. 2013. Intradermal adipocytes mediate fibroblast recruitment during skin wound healing. Development 140: 1517-1527.

Schmidt-Ullrich R, Paus R. 2005. Molecular principles of hair follicle induction and morphogenesis. Bioessays 27: 247-261.

Seifert AW, Kiama SG, Seifert MG, Goheen JR, Palmer TM, Maden M. 2012a. Skin shedding and tissue regeneration in African spiny mice (Acomys). Nature 489: 561-565.
Seifert AW, Monaghan JR, Voss SR, Maden M. 2012b. Skin regeneration in adult axolotls: A blueprint for scar-free healing in vertebrates. PLoS ONE 7: e32875.

Sennett R, Rendl M. 2012. Mesenchymal-epithelial interactions during hair follicle morphogenesis and cycling. Semin Cell Dev Biol 23: 917-927.

Shah M, Foreman DM, Ferguson MW. 1995. Neutralisation of TGF- $\beta 1$ and TGF- $\beta 2$ or exogenous addition of TGF$\beta 3$ to cutaneous rat wounds reduces scarring. J Cell Sci 108: $985-1002$.

Snippert HJ, Haegebarth A, Kasper M, Jaks V, van Es JH, Barker N, van de Wetering M, van den Born M, Begthel H, Vries RG, et al. 2010. Lgr6 marks stem cells in the hair follicle that generate all cell lineages of the skin. Science 327: 1385-1389.

So K, McGrouther DA, Bush JA, Durani P, Taylor L, Skotny G, Mason T, Metcalfe A, O’Kane S, Ferguson MW. 2011. Avotermin for scar improvement following scar revision surgery: A randomized, double-blind, within-patient, placebo-controlled, phase II clinical trial. Plast Reconstr Surg 128: $163-172$.

Somasundaram K, Prathap K. 1970. Intra-uterine healing of skin wounds in rabbit foetuses. J Pathol 100: 81-86.

Soo C, Beanes SR, Hu FY, Zhang X, Dang C, Chang G, Wang Y, Nishimura I, Freymiller E, Longaker MT, et al. 2003. Ontogenetic transition in fetal wound transforming growth factor- $\beta$ regulation correlates with collagen organization. Am J Pathol 163: 2459-2476.

Sopher D. 1972. The response of rat fetal membranes to injury. Ann R Coll Surg Engl 51: 240-249.

Takeo M, Chou WC, Sun Q, Lee W, Rabbani P, Loomis C, Taketo MM, Ito M. 2013. Wnt activation in nail epithelium couples nail growth to digit regeneration. Nature 499: $228-232$.

Taylor G, Lehrer MS, Jensen PJ, Sun TT, Lavker RM. 2000. Involvement of follicular stem cells in forming not only the follicle but also the epidermis. Cell 102: 451-461.

Tumbar T, Guasch G, Greco V, Blanpain C, Lowry WE, Rendl M, Fuchs E. 2004. Defining the epithelial stem cell niche in skin. Science 303: 359-363.

Wulff BC, Parent AE, Meleski MA, DiPietro LA, Schrementi ME, Wilgus TA. 2012. Mast cells contribute to scar formation during fetal wound healing. I Invest Dermatol 132: $458-465$.

Yokoyama H, Maruoka T, Aruga A, Amano T, Ohgo S, Shiroishi T, Tamura K. 2011. Prx-1 expression in Xenopus laevis scarless skin-wound healing and its resemblance to epimorphic regeneration. J Invest Dermatol 131: 2477-2485.

Zhang YV, Cheong J, Ciapurin N, McDermitt DJ, Tumbar T. 2009. Distinct self-renewal and differentiation phases in the niche of infrequently dividing hair follicle stem cells. Cell Stem Cell 5: 267-278.

Zhao W, Neufeld DA. 1995. Bone regrowth in young mice stimulated by nail organ. J Exp Zool 271: 155-159. 


\section{$\&_{\mathrm{CSH}}^{\infty} \&$ Cold Spring Harbor

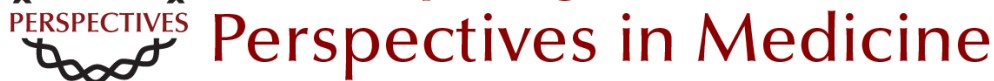

\section{Wound Healing and Skin Regeneration}

Makoto Takeo, Wendy Lee and Mayumi Ito

Cold Spring Harb Perspect Med 2015; doi: 10.1101/cshperspect.a023267

Subject Collection The Skin and Its Diseases

Melanoma: Clinical Features and Genomic Insights

Elena B. Hawryluk and Hensin Tsao

Wound Healing and Skin Regeneration

Makoto Takeo, Wendy Lee and Mayumi Ito

The Dermal Papilla: An Instructive Niche for

Epithelial Stem and Progenitor Cells in

Development and Regeneration of the Hair Follicle Bruce A. Morgan

Immunology and Skin in Health and Disease Jillian M. Richmond and John E. Harris

Desmosomes: Regulators of Cellular Signaling and Adhesion in Epidermal Health and Disease Jodi L. Johnson, Nicole A. Najor and Kathleen J. Green

Markers of Epidermal Stem Cell Subpopulations in Adult Mammalian Skin Kai Kretzschmar and Fiona M. Watt

Psoriasis Paola Di Meglio, Federica Villanova and Frank O. Nestle

Cell Therapy in Dermatology Gabriela Petrof, Alya Abdul-Wahab and John A. McGrath
Modeling Cutaneous Squamous Carcinoma

Development in the Mouse

Phillips Y. Huang and Allan Balmain

Natural and Sun-Induced Aging of Human Skin Laure Rittié and Gary J. Fisher

Advanced Treatment for Basal Cell Carcinomas Scott X. Atwood, Ramon J. Whitson and Anthony E. Oro

Epidermal Polarity Genes in Health and Disease Frederik Tellkamp, Susanne Vorhagen and Carien M. Niessen

Induced Pluripotent Stem Cells in Dermatology:

Potentials, Advances, and Limitations Ganna Bilousova and Dennis R. Roop

The Genetics of Human Skin Disease Gina M. DeStefano and Angela M. Christiano

p53/p63/p73 in the Epidermis in Health and Disease

Vladimir A. Botchkarev and Elsa R. Flores

Diversification and Specialization of Touch

Receptors in Skin

David M. Owens and Ellen A. Lumpkin

For additional articles in this collection, see http://perspectivesinmedicine.cshlp.org/cgi/collection/ 